\title{
Learning Food Safety, Proper Nutrition and (COVID-19)
}

Moustafa A.El-Shenawy

Dept. Food Sci. \& Bacteriology, Division of Food Industry and Nutrition, National Research Center, Cairo - Egypt.

*Corresponding author: Moustafa A.El-Shenawy, Dept. Food Sci. \& Bacteriology, Division of Food Industry and Nutrition, National Research Center, Cairo - Egypt.

Received date: June 09, 2020; Accepted date: July 28, 2020; Published date: August 03, 2020.

Citation: Moustafa A.El-Shenawy, (2020) Learning Food Safety, Proper Nutrition and (COVID-19). J. Nutrition and Food Processing, 3(2); DOI: $10.31579 / 2637-8914 / 027$

Copyright: (C) 2020 Moustafa A.El-Shenawy, This is an open-access article distributed under the terms of the Creative Commons Attribution License, which permits unrestricted use, distribution, and reproduction in any medium, provided the original author and source are credited.

\section{Abstract}

There is not yet accurate and confirmed information about that emerging coronavirus (Covid-19), or about reaching an appropriate treatment that eliminates it. Till this moment, the global medical authorities have agreed that simple prevention methods are the application of precautionary measures, especially in the absence of a preventive serum, which prompted many questions about the food handling/safety issues you should deal with during this crisis. Moreover, the proper nutrition is vital and important. Thus eating a balanced diet lead to healthier and stronger immunity and less exposed to the risk of chronic and infectious diseases including viral infection. This mini-review clarifies the rules that dealing with your purchases of food in order to be safe and not be a source for the spread of the virus during this Pandemic outbreak. Also, it sheds light on types of healthy stock foods you can buy and some of the nutritional rules that must be followed in order to raise immunity, which is a key factor to resist the virus.

Keywords: Food Safety; Proper Nutrition; COVID-19

\section{Introduction}

The Centers for Disease Control and Prevention (CDC) and the Food and Drug Administration (FDA), and the World Health Organization (WHO) have unanimously agreed that there is no information about the transmission of the virus through food, but rather that, it is transmitted through flying mist from the person's nose or mouth. This flying mist/spray may directly reach another person and transmit the infection, after penetrating the upper respiratory barrier, and/or it may reach the different surfaces and upon contact with it and placing the hand on the mouth/eyes in face the infection is transmitted. Thus, this spray may reach the food (raw or cooked), this way, and it can stay in if it is not cleansed of disinfected. From this point the different foods need a cautions and methods to ensure their safety to avoid any possibility of infection.

This mini review, highlights the basic principles/methods used to disinfect various foods to prevent the emerging corona virus. Also, it sheds light on types of healthy stock foods you can buy as well as the main nutritional rules that must be followed in order to raise human immunity, as the main factor to resist the viral infection.

\section{General hygiene statements}

Frist of all, As a general trend, Attention to general hygiene must be taken to wash hands with soap and water, for a period of not less than 20 seconds, before preparing food, taking into account cleaning the kitchen surfaces and cupboard knobs and inserting the refrigerator with household antiseptics, to get rid of attached viruses, that can move to food by contact.

Care must be taken to wash foods well before eating, even they were washed before, and this applies to fruits that do not eat their outer shell, such as oranges and watermelons, where they must be rinsed with running water, to get rid of dirt and germs accumulated on its external surface, taking into account the use of a soft brush with foods with a thin shell Like strawberries.

\section{The rules that dealing with your purchases of food}

\section{1- Shop safely}

When go shopping, care must be taken to leave a distance of at least 6 feet between you and others, taking into account wearing a medical mask, to prevent cross infection due to crowding. It is preferable to wipe/disinfect the shopping cart handle well and wear rubber gloves before choosing/purchasing the products, because it may have been previously touched by a person infected with the virus.

\section{2- Sterilizing the main plastic bags}

When returning home, after shopping, care must be taken to wash hands thoroughly with running water and soap, and then sterilize the main plastic bags, that contain your purchases, with a towel moistened with a household disinfectant, such as chlorine solution, to eliminate viruses and bacteria sticking to its outer surface.

\section{How to deal with your purchased items.}

\section{A- Cans, packaged and bagged foods}

All items purchased from the market, such as packaged and bagged foods, as well as cans, are shown on the shelves and subjected for public up to days, which may be exposed to flying mist from any person who is infected/carrier of the virus. Because Information has been conflicting about how long the virus stays on the surfaces which may reach three days and possibly more, So we must follow some guidelines for disinfection, which is the following: 1- bags loaded with food supplies must be left on the outside of the home or , out of reach ,for a period of three days.2- After this period has ended, the hands should be thoroughly washed with soap 
and water, and the items should be removed from the bags that dumped in the garbage, and the items should be placed on a pre-cleaned surface.3Disinfection of bags that carry grains and other foods coated in sheets or carton with an antiseptic containing $70 \%$ alcohol and left to dry, and it is necessary to ensure that the disinfectant does not reach inside.4- Do not place items in the refrigerator for other three days if they are not subject to damage because viruses often activate and remain on surfaces in a cool, humid environment.5-Cleansing the surface where the items were placed on well, then wash hands with soap and water.

\section{B- Fruits and vegetables}

Fruits and vegetables are exposed to air, which may expose them to the arrival of bacteria and viruses. Therefore, they must be cleaned and sterilized in a proper way to ensure their safety before eating them. Therefore, the following must be done: 1- Drain fruits in clean, washed dishes with soap and water. 2- Dispose of bags in which the fruits were in the trash, and wash hands thoroughly with soap and water. 3- Wash each fruit well under running water, then transfer to a clean bowl. 4- After finishing washing all the fruits, put a few drops of vinegar and water in the bowl and leave it for 15 minutes. 5- Re-wash the fruits with water to get rid of the smell of vinegar.

\section{C- Meat}

Although, up till now, there is no information that indicate the possibility of human-to-animal transmission of the virus, however veterinary quarantine measures should be applied on your purchased meat to limit the transmission of the virus since the product can be contaminated from human during its preparation for sale. Here are some standards and number of notes that must be followed:-

1- Separate different types of meat from each other, and also separate them from vegetables, fruits, and other types of food. 2- Clean the meat before placing it in the refrigerator to ensure its safety.3- The meat is thoroughly washed with running water and any suspended deposits are removed. 4-Red meat needs vinegar, salt and lemon to get rid of any sediment and ensure its safety. 5- The meat is placed in the designated and clean storage bags in the cooler after finishing the cleaning process. 6- Meat should be cleaned in containers other than those used for other purposes.7- Clean surfaces well after finishing and washing hands with soap and water.

\section{D- The bread}

The necessary standards must be followed when purchasing bread to avoid catching any infection during purchase, and baked goods must be exposed to fire directly because high temperatures can kill any viruses.

\section{The healthy stock foods you can buy.}

The new Corona virus has locked up a third of the world's population in their homes, and not get out of it, as some countries have had to enforce the curfew in the streets and squares, as a precautionary measure to prevent the spread of Covid-19. Therefore purchase quantities of stock foods sufficient for this critical period is a must. Here is a list of the most important non-perishable healthy foods that are recommended to be present at home during the ban period.

\section{1- Beans}

Beans are legumes rich in nutrients beneficial to human health, such as fiber, vegetable protein, magnesium, vitamin B, manganese, iron, phosphorous, zinc, and copper. Beans can be stored at normal room temperature, for up to two years or more, without being damaged, and for this reason, they are among the foods that are recommended to be present at home during this critical period.

\section{2- Dried fruits and vegetables}

Fresh fruits and vegetables have a short shelf life, so dried fruits and vegetables can be relied on as foods of high nutritional value. They have a validity of one year. Proper storage guidelines must be observed, so that they are kept in sealed packages, to keep them from being damaged.

\section{3- Canned fish}

Although fresh fish have a high nutritional value, they are rapidly spoiled, and therefore, canned fish, such as tuna, must be relied on because they can be kept for a long period of time, up to $3-5$ years, without spoiling, as long as it is stored correctly.

\section{4- Nuts}

It is one of the foods that can be stored for a long time, up to 4 months or more, especially if it is kept in a non-humid environment. Nuts are distinguished by their high content of nutrients that a person needs, but the source from which they will be purchased must be taken into account, and to make sure of their validity.

\section{The main nutritional rules that must be followed to raise immunity}

Proper nutrition and abundance of drinking water are vital and important. Those who eat a balanced diet are more likely to have healthier, stronger immune systems and less at risk of developing chronic and infectious diseases. So you should eat a variety of fresh and unprocessed foods every day to get the vitamins, minerals, dietary fiber, protein and antioxidants your body needs.

Here are the main rules you should apply to raise your immunity.

\section{Eat fresh, unprocessed foods every day}

Eat fruits, vegetables, legumes (such as lentils and beans), nuts and whole grains (such as unprocessed corn, millet, oats, wheat, brown rice, starchy tuber or roots such as potatoes, yams, taro, or cassava) and animal-source foods (such as meat, fish, eggs, and milk).

Daily, eat: 2 cups of fruit (4 servings), 2.5 cups of vegetables ( 5 servings), 180 grams of cereals, 160 grams of meat and beans (red meat can be eaten once to twice a week, and poultry 2-3 times a week) .

For snacks, choose raw vegetables and fresh fruit, rather than foods rich in sugar, fat or salt.

Avoid overcooking vegetables and fruits, as this may lead to the loss of important vitamins.

When using canned or dried fruits and vegetables, choose those that do not contain salt or sugar.

\section{Drink enough water every day}

Water is essential for life. It transports nutrients and compounds in the blood, regulates body temperature, eliminates waste, reduces and facilitates joint mobility.

Drink 8-10 glasses of water every day.

Water is the best option, but drinks, fruits and other vegetables that contain water can also be consumed, for example lemon juice (diluted in water and unsweetened), tea, and coffee. But be careful not to consume a lot of caffeine, and avoid sweetened fruit juices, juices, fruit juice concentrates, soft drinks and refreshing drinks as they all contain sugar.

\section{Eat moderate amounts of fats and oils}

Eat unsaturated fats (such as found in fish, avocados, nuts, olive oil, soybeans, canola, sunflower, and corn oils) instead of saturated fats (such as found in fatty meats, butter, palm oils, coconut, cream, cheese, ghee, and lard). 
Choose white meat (such as poultry) and fish, which are generally low in fat, instead of red meat. Avoid processed meats, as they are high in fat and salt.

Eat milk and its low-fat or low-fat products whenever possible. Avoid eating trans-fats which is often found in processed foods, fast food, snacks, fried foods, frozen pizza, pies, cakes, ghee, and foods that are greased on bread.

\section{Eat less salt and sugars}

When cooking and preparing food, reduce the amount of sodium-rich salt and spices (such as soy sauce and fish sauce). Reduce your daily salt intake to less than 5 grams (about one teaspoon), and eat iodized salt. Avoid foods rich in salt and sugars (such as snacks). Reduce the intake of soft drinks or soda and other sugary drinks (such as fruit juices, concentrated juices, concentrated syrups, flavored milk, and yogurt drinks). Choose fresh fruits instead of sweetened snacks such as biscuits, cakes and chocolate.

\section{Avoid eating outside}
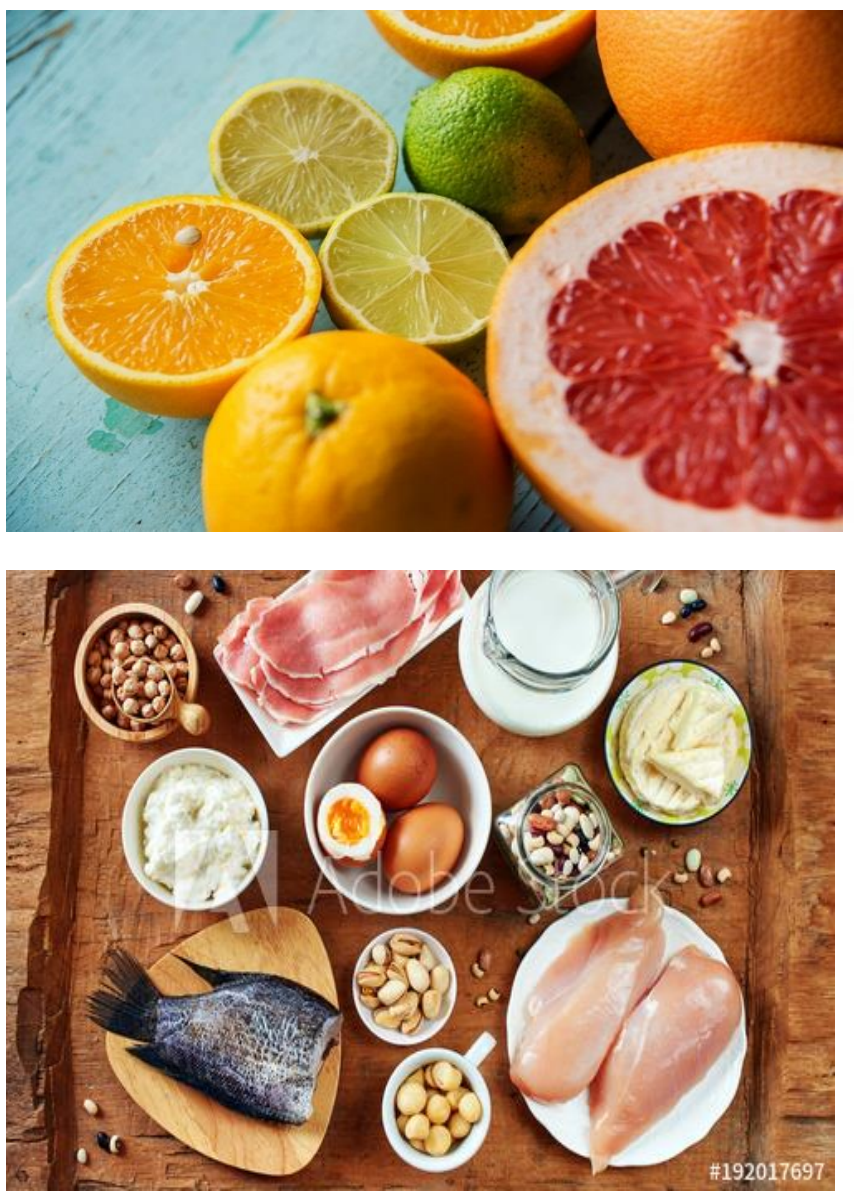

Eating at home to reduce contact rate and reduce your exposure to the emerging coronavirus (Covid 19). We recommend that you keep a distance of at least one meter between you and anyone who coughs or sneezes. This is not always possible in crowded social places like restaurants and cafes. Drops of infected spray may fall on the surfaces and hands of people (such as clients and employees), and due to the large number of arrivals and departures, it will not be possible to know whether the hands are washed regularly and sufficiently, and that the surfaces are cleaned and sanitized fast enough.

\section{Seeking psychological and social counseling and support}

Although proper nutrition and abundance of drinking water improve health and immunity, they are not a panacea for disease prevention. People with chronic diseases who are suspected or confirmed to be infected with the virus need to support their mental health and support their diet to ensure their health. So, seek psychosocial advice and support from appropriately trained healthcare professionals as well as from community counselors and peers.
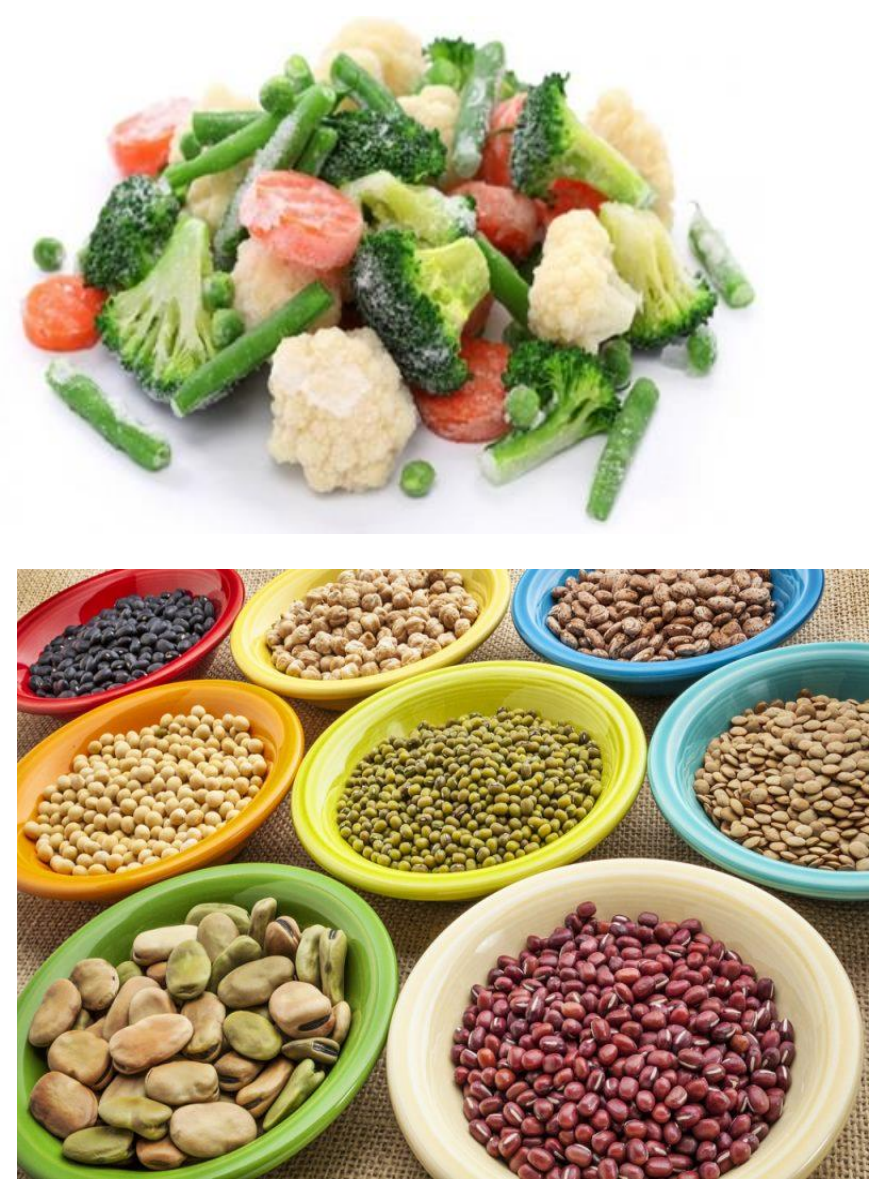